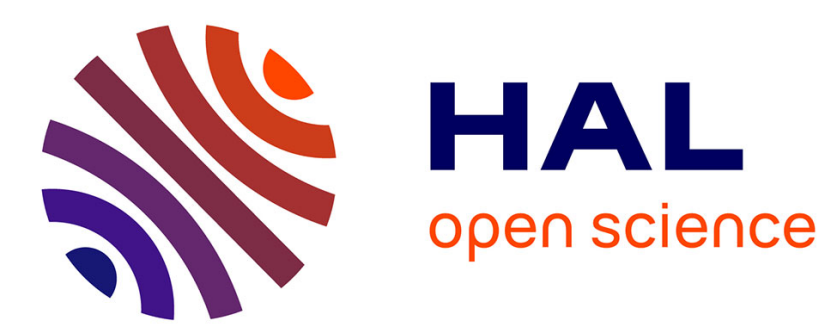

\title{
Digital holographic interferometry at F4-ONERA hypersonic wind tunnel
}

J.M. Desse, J. Soutadé, P. Viguier, Pascal Picart, M. Ferrier, L. Serre

\section{To cite this version:}

J.M. Desse, J. Soutadé, P. Viguier, Pascal Picart, M. Ferrier, et al.. Digital holographic interferometry at F4-ONERA hypersonic wind tunnel. 16th International Symposium on Flow Visualization (ISFV16 2014), Jun 2014, OKINAWA, Japan. hal-01079254

\section{HAL Id: hal-01079254 \\ https://hal.science/hal-01079254}

Submitted on 31 Oct 2014

HAL is a multi-disciplinary open access archive for the deposit and dissemination of scientific research documents, whether they are published or not. The documents may come from teaching and research institutions in France or abroad, or from public or private research centers.
L'archive ouverte pluridisciplinaire HAL, est destinée au dépôt et à la diffusion de documents scientifiques de niveau recherche, publiés ou non, émanant des établissements d'enseignement et de recherche français ou étrangers, des laboratoires publics ou privés. 


\title{
DIGITAL HOLOGRAPHIC INTERFEROMETRY AT F4-ONERA HYPERSONIC WIND TUNNEL
}

\author{
J.M. DESSE ${ }^{1, c}$, J. SOUTADE ${ }^{2}$, P. VIGUIER ${ }^{2}$, P. PICART ${ }^{3}$, M. FERRIER ${ }^{4}$, L. SERRE ${ }^{4}$ \\ ${ }^{1}$ Office National d'Etudes et Recherches Aérospatiales, The French Aerospace Lab, Lille, 59045, France \\ ${ }^{2}$ Office National d'Etudes et Recherches Aérospatiales, The French Aerospace Lab, Fauga-Mauzac, 31410, France \\ ${ }^{3}$ Laboratoire d'Acoustique de l'Université du Maine, Le Mans, 72085, France \\ ${ }^{4}$ Office National d'Etudes et Recherches Aérospatiales, The French Aerospace Lab, Palaiseau, 91761, France \\ ${ }^{\mathrm{c} C}$ Corresponding author: Tel.: +33320496940; Fax: +33320496953; Email: Jean-Michel.Desse.@ onera.fr
}

KEYWORDS:

Main subjects: aerodynamics, hypersonic flow

Fluid: ionized gas

Visualization method(s): digital holography, schlieren technique

Other keywords: luminous interference, gas density measurement

\begin{abstract}
The feasibility of digital holographic interferometry using a CW laser @ 532 nm and a Photron APX camera is demonstrated in the F4-ONERA hypersonic wind tunnel. This optical technique is used to point out the possible combustion of a small hydrogen jet in the shock layer developed on the model. The optical technique generates in the field under analysis, interference micro-fringes which are used as carrier spatial frequency. The field of optical thickness is obtained with a FFT2D post processing. Then, if the flow is assumed to be twodimensional, the map of the refractive index can be estimated. The adjustment of the optical bench is easy to implement and the results obtained with this technique can be used for several interesting comparisons: from the phase difference maps, the holographic images and schlieren images in the $x$ and $y$ directions can be recalculated, which is not possible with a conventional schlieren optical setup. In addition, if the characteristics of the ionized gas are well known, the value of the gas density upstream and in the shock layer can be estimated.
\end{abstract}

\section{Introduction}

One of optical methods developed over several years at ONERA for the gas density measurement is based on holographic interferometry, using one or more wavelengths as luminous light source. Firstly developed with panchromatic holographic plates used in transmission or reflection, the technique has evolved to become a fully digital technology based on the generation of spatial carrier frequencies analyzed by 2D Fast Fourier Transform [1].

The first tests performed in an ONERA transonic wind tunnel have resulted in analyzing the unsteady flow downstream of a circular cylinder located in the test section for subsonic and transonic Mach numbers. Convincing results can be found in Desse and Picart [2] and the implementation of digital holographic interferometry has been considered in several ONERA wind tunnels among which the F4 hypersonic Fauga-Mauzac facility. Here, the difficulty lies in the fact that, as the gas density of the upstream flow $\rho_{0}$ is very low (close to $10^{-4}$ or $10^{-5} \mathrm{~kg} / \mathrm{m}^{3}$ ), the refractive index $\mathrm{n}_{0}$ related to $\rho_{0}$ is also very small, and thus, the differences in refractive index $\left(n-n_{0}\right)$ are even smaller and more difficult to measure. 
The optical technique used up to now in the F4 hypersonic wind tunnel is a schlieren technique using a schlieren knife or a colored mask. The refractive index gradients in the direction perpendicular to the knife edge can be visualized. Thus, if the knife edge is located vertically in the focusing spot beam, horizontal gradients in the refractive index will be highlighted. This technique shows its limits when the refractive index gradients are very low. In this case, it is preferable to use optical methods which directly visualize the refractive index and not the refractive index gradient. Holography and holographic interferometry are suitable to measure the absolute value of the refractive index. Surget et al. [3] and Surget and Dunet [4] exploited holographic interferometry in F4 hypersonic facility, in which the crossing number of the test section was multiplied by 2,3 or 4 , which increased in the same ratio the measurement sensitivity. A comparison between the schlieren technique and transmission holographic interferometry using a holographic plate was shown. The difficulty in transmission holographic interferometry developed by Surget was that the signal-to-noise ratio increased with the crossing number, which makes the quantitative processing of interferograms difficult. Otherwise, the idea which consists in crossing the flow several times to increase the measurement sensitivity is an idea that can be applied to digital holographic interferometry based on the principle described by Surget and Dunet.

This document describes therefore the feasibility of digital monochromatic holographic interferometry performed in F4 hypersonic wind tunnel on the flow around a wedge at Mach 10 and also the improvement opportunities of the optical setup that has been implemented.

\section{Theoretical background of digital holography}

Hologram analysis performed by direct and inverse two-dimensional FFT algorithms is very well suited for the reconstruction of transparent phase objects. For any wavelength $\lambda$, the image plane hologram recorded can be expressed as:

$H_{\lambda}(x, y)=O_{0}(x, y)+R(x, y) O^{*}(x, y)+R^{*}(x, y) O(x, y)$

where $O_{0}(x, y)$ and $R(x, y)$ are the zero order diffraction and the reference wave respectively and $O=b_{\lambda} \exp \left(i \varphi_{\lambda}\right)$ is the object wave. For convenience, $R(x, y)$ can be represented with unitary amplitude and zero phase. In the case of in-line holography, computation by Fourier transform gives a broad spectrum centered at the zero spatial frequency. So, no pertinent information can be extracted from such a spectrum. Consider now off-axis holography in which a spatial carrier is introduced along $x$ or $y$ or along the two directions. In a general case, $2 \pi\left(u_{\lambda} x+v_{\lambda} y\right)$ is the spatial carrier modulation along $x y$, the hologram becomes:

$H_{\lambda}(x, y)=O_{0}(x, y)+O(x, y) \exp \left[2 i \pi\left(u_{\lambda} x+v_{\lambda} y\right)\right]+O^{*}(x, y) \exp \left[-2 i \pi\left(u_{\lambda} x+v_{\lambda} y\right)\right]$

By developing complex exponentials one obtains:

$$
H_{\lambda}(x, y)=O_{0}(x, y)+b_{\lambda}(x, y) \exp \left[i \varphi_{\lambda}(x, y)\right] \exp \left[2 i \pi\left(u_{\lambda} x+v_{\lambda} y\right)\right]+b_{\lambda}(x, y) \exp \left[-i \varphi_{\lambda}(x, y)\right] \exp \left[-2 i \pi\left(u_{\lambda} x+v_{\lambda} y\right)\right]
$$

Fourier transform of Eq. (3) gives:

$$
\tilde{H}_{\lambda}(u, v)=A_{\lambda}(u, v)+C_{\lambda}\left(u-u_{\lambda}, v-v_{\lambda}\right)+C_{\lambda}^{*}\left(u+u_{\lambda}, v+v_{\lambda}\right)
$$


where $C_{\lambda}(u, v)$ and $A_{\lambda}(u, v)$ are respectively the Fourier transform of $b_{\lambda}(x, y) \exp \left[i \varphi_{\lambda}(x, y)\right]$ and $O_{0}(x, y)$. If the spatial frequencies $u_{\lambda}$ and $v_{\lambda}$ are well chosen, the three orders are well separated in the Fourier plane. Applying a binary mask around the frequency $+u_{\lambda},+v_{\lambda}$, respectively of width $\Delta u$ and $\Delta \mathrm{v}$, allows the extraction of the object optical phase $\varphi_{\lambda}(x, y)$. Inverse Fourier transform applied to the selected region gives an estimation of the object complex amplitude:

$\hat{c}_{\lambda}(x, y) \cong\left\{b_{\lambda}(x, y) \exp \left[i \varphi_{\lambda}(x, y)\right] \exp \left[2 i \pi\left(u_{\lambda} x+v_{\lambda} y\right)\right]\right\} * h(x, y)$

where * means convolution and $h(x, y)$ is the impulse response corresponding to the filtering applied in the Fourier domain. It is such that

$h(x, y)=\Delta u \Delta v \exp \left[2 i \pi\left(u_{\lambda} x+v_{\lambda} y\right)\right] \operatorname{sinc}(\pi \Delta u x) \operatorname{sinc}(\pi \Delta v y)$

After the filtering process, a biased optical phase can be estimated from relation (5):

$\xi_{\lambda}^{R}(x, y)=\varphi_{\lambda}(x, y)+2 \pi u_{\lambda} x+2 \pi v_{\lambda} y=\arctan \left(\frac{\mathfrak{I}_{m}[\hat{c}(x, y)]}{\mathfrak{R}_{e}[\hat{c}(x, y)]}\right)$

and the amplitude of the fundamental harmonic at wavelength $\lambda$ by:

$\hat{b}_{\lambda}(x, y)=\sqrt{\mathfrak{I}_{m}^{2}[\hat{c}(x, y)]+\mathfrak{R}_{e}^{2}[\hat{c}(x, y)]}$

In equations $(7,8), \mathfrak{I}_{m}[\ldots]$ and $\mathfrak{\Re}_{e}[. .$.$] mean imaginary and real part of complex value.$

Note that the suppression of the spatial carrier can be obtained by multiplying Eq. (5) by $\exp \left[2 i \pi\left(u_{\lambda} x+v_{\lambda} y\right)\right]$. When the test object is modified, for example by heating or with a flow circulation, this induces a modification in the refractive index along the probe beam and thus this modifies the optical path and then the optical phase. At any wavelength $\lambda$, recording a new hologram $H_{\lambda}(x, y)$ leads to the computation of phase $\xi_{\lambda}^{P}(x, y)=\varphi_{\lambda}(x, y)+\Delta \varphi_{\lambda}(x, y)+2 \pi u_{\lambda} x+2 \pi_{\lambda} y$. The phase change between the two states of the object is simply obtained by computing $\Delta \varphi_{\lambda}(x, y)$. In a setup such that the measurement beam crosses twice the tested region, the optical path difference due to the phenomena under interest is given by:

$\delta=\frac{\lambda}{4 \pi} \Delta \varphi_{\lambda}$

\section{Experimental setup}

\subsection{F4 wind tunnel}

The ONERA F4 wind tunnel was commissioned during the course of the ESA HERMES program in the early 1990s, and has since been extensively used to test various reentry vehicles, for both terrestrial and Martian atmosphere. 
The F4 wind tunnel is a hot shot type facility, meaning that the operating conditions are obtained by heating the test gas with an intense electric arc in a chamber of 10 to 151 volume initially pressurized at ambient temperature. This chamber is a cylindrical tank whose lateral sides are the electrodes; the electrode shape induces a rotation of the arc. This enhances the efficiency of the heating process and reduces the erosion of the electrode head. The electrode bodies are thermally and electrically insulated from the chamber. Two exits connect the chamber downstream to the nozzle and upstream to the quick discharge exhaust tank.

The energy is delivered by an impulse generator, at a power of up to $150 \mathrm{MW}$ for several tens of milliseconds. The settling-chamber pressure Pi can be as high as $1 \mathrm{kbar}$, and the total enthalpy can be as high as $18 \mathrm{MJ} / \mathrm{kg}$ for air. A run duration of up to $400 \mathrm{~ms}$ can be achieved.

\subsection{Model and optical setup}

The aim of this test is to determine if the digital holographic interferometry can be used to visualize the injection of a small jet of hydrogen at 32 bar on a flat plate at a $20^{\circ}$ incidence angle. If the jet is correctly visualized with the optical technique, information on the combustion process of the hydrogen jet in the shock layer can be obtained. In this test, the plate is $260 \mathrm{~mm}$ wide and the injection is located at $117 \mathrm{~mm}$ from the leading edge a shown in Fig. 1.

The optical setup shown in Fig. 2 looks like a Michelson interferometer where a reference wave can interfere with an object wave into CCD or CMOS sensor. A DPSS laser $(\lambda=532 \mathrm{~nm})$ is used as the luminous light source of the interferometer and a beam splitter cube separates the incident beam into the reference wave, which is returned by the concave mirror and the object which crosses twice the test section. A spatial filter (x60) equipped with a small hole of $25 \mu \mathrm{m}$ is located at the focal length of the large achromatic lens (FL $800 \mathrm{~mm}$ ) so that the test section may be illuminated with a parallel beam. A flat mirror located just behind the test section returns the beam into the beam splitter cube and onto the camera sensor. The second small achromatic lens (FL $75 \mathrm{~mm}$ ) forms the image magnification on the sensor.

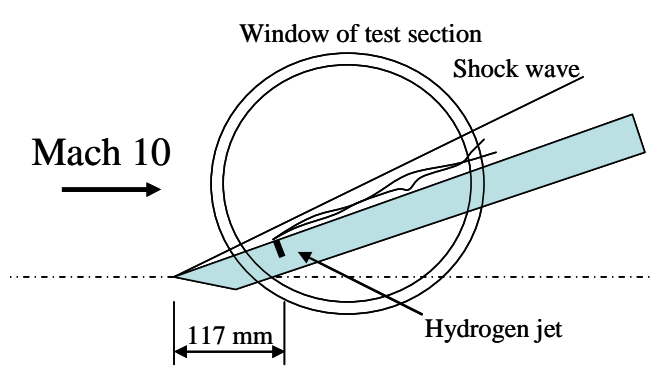

Fig. 1 Schematic model.

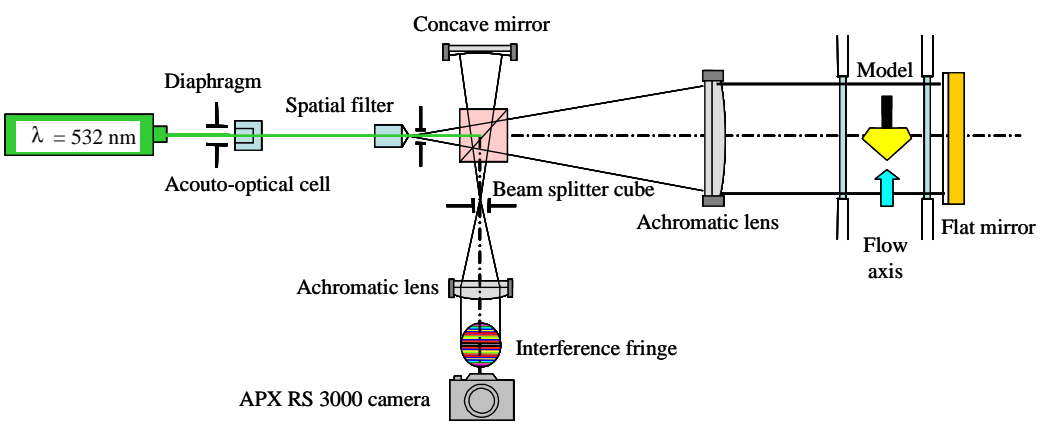

Fig. 2 Implementation of digital holographic interferometer.

\section{Obtained results}

As the test lasts about 200 milliseconds, the interferograms are recorded using a Photron APX RS 3000 camera at the framing rate of 3,000 images per second ( $333.33 \mu$ s between each interferogram). The exposure time of each image fixed by the camera is $1 \mu \mathrm{s}$ and the camera is triggered by a TTL signal generated $215 \mathrm{~ms}$ before the beginning of the burst. The recording procedure is as follows: 
- the interferometer is adjusted with a uniform background color. Then, the concave mirror is tilted to introduce the reference micro-fringes which will constitute the spatial carrier frequency.

- then, the reference interferogram is recorded without flow in the test section.

- finally, the camera triggered with the run records the measurement interferograms at a high framing rate. Here, as the camera is triggered a few hundred microseconds before the beginning of the burst, the first images of the film can be easily used as reference images.

Fig. 3a shows two images of interference micro-fringes recorded with and without flow (reference and measurement). In the enlarged part of the shock layer located just above the injection, the disturbance of the interference micro-fringes can be observed, both by the shock wave and by the hydrogen jet. The first analysis step consists in computing FFT2D spectra of the reference and measurement images. Fig. $3 \mathrm{~b}$ shows the results obtained in the four quadrants of the spectrum. The +1 and -1 symmetrical orders with $\mathrm{u}_{0}$ and $\mathrm{v}_{0}$ coordinates corresponding to carrier frequencies generated by the optical setup - and the central zero-order are very well identified. The filtering step consists in applying a window around the +1 order and in reconstructing the amplitude and phase of the hologram with the information contained around the +1 order.

Considering the values of the spatial carrier frequencies, $\mathrm{u}_{0}$ and $\mathrm{v}_{0}$ are respectively equal to $15 \mathrm{~mm}^{-1}$ and $10 \mathrm{~mm}^{-1}$ that gives a resolution of about 10 to 15 fringes per $\mathrm{mm}$ in the plane of the CCD camera. As the optical setup magnification is here given by the ratio between the diameter of the large achromatic lens $(200 \mathrm{~mm})$ and the sensor size $(17 \mathrm{~mm})$, the resolution in the test section is equal to $0.76 \mathrm{~mm}$ along the $\mathrm{x}$ axis and $1.14 \mathrm{~mm}$ along the $\mathrm{y}$ axis. These values may seem high, but they are mainly related to the pixel size of the camera that was used in this study of feasibility.
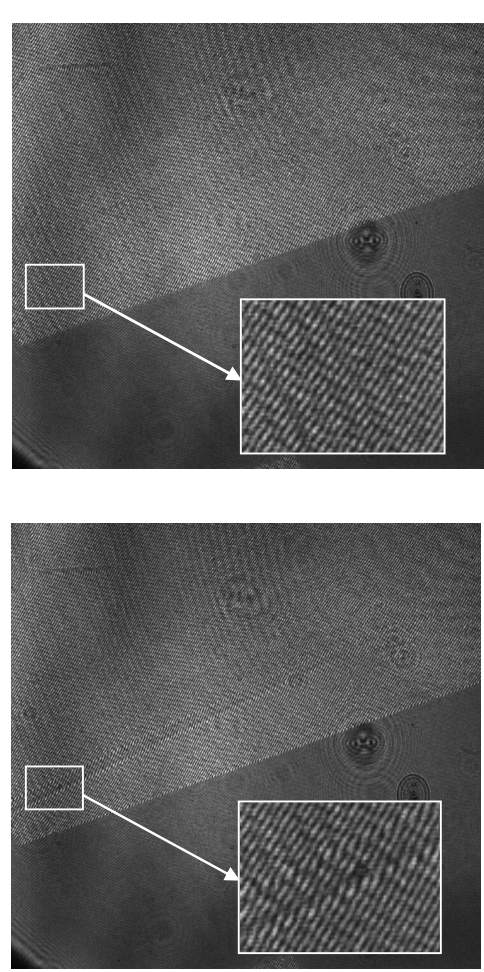

a) Interferograms
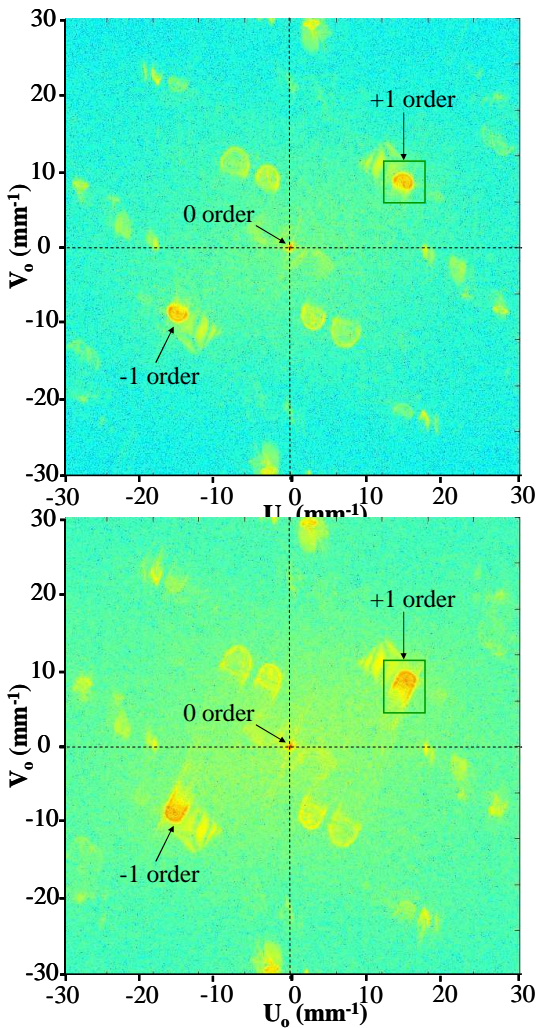

b) Fourier spectra
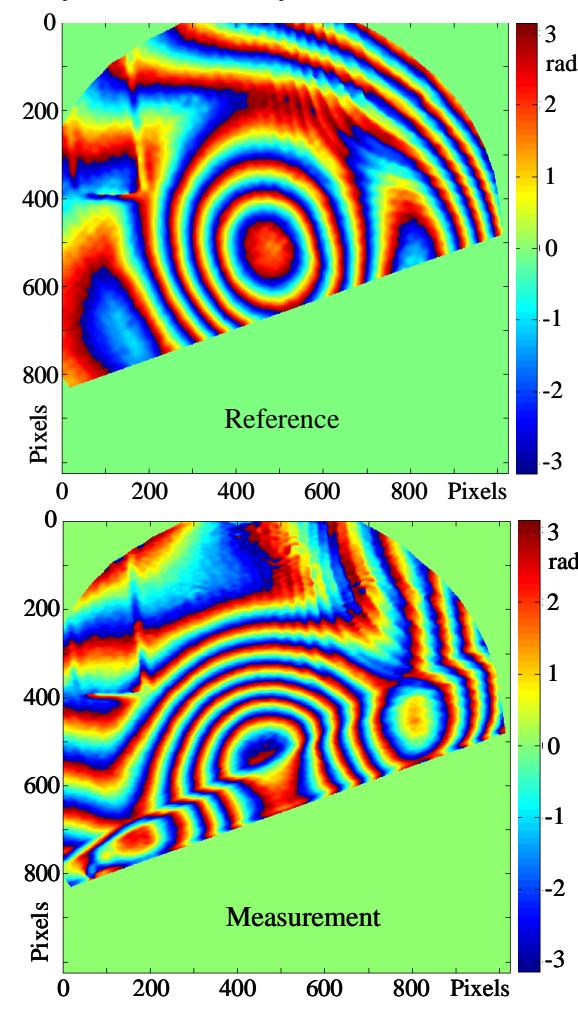

c) Modulo 2p phase map

Fig. 3 Reference and measurement interferograms analysis 
Once the filtering window determined, the reference and measurement phase maps shown in Fig. $3 \mathrm{c}$ are calculated. It should be noted that the phase maps can also be calculated by saving the spatial carrier frequency. As the phases are calculated modulo $2 \pi$, an unwrapping process has to be applied to obtain the phase map. Fig. 4a shows the phase processing (unwrapped) for the measurement. From the phase map of the phenomenon, the optical thickness map can easily be calculated and Fig. $4 \mathrm{~b}$ shows an example where the optical thickness varies between -0.4 and 0.4 microns. After having analyzed all the interferograms of the sequence, we noted that no evidence of combustion of the hydrogen jet in the shock layer can be pointed out. Knowing the phase difference of each frame, the luminous intensity of the interference fringes, which would have been visualized if a technique using transmission or reflection plate holography had been implemented, can be calculated. Fig. 4c shows the interferogram computed from the phase difference maps. The subtraction of an initial optical thickness is equivalent to recording interferogram with a uniform background color, which leads to a fine visualization of the shock wave location and of the small hydrogen jet injection.

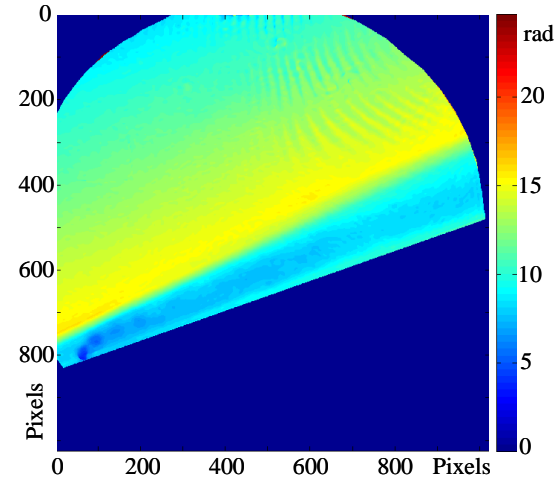

a) Unwrapped phase difference

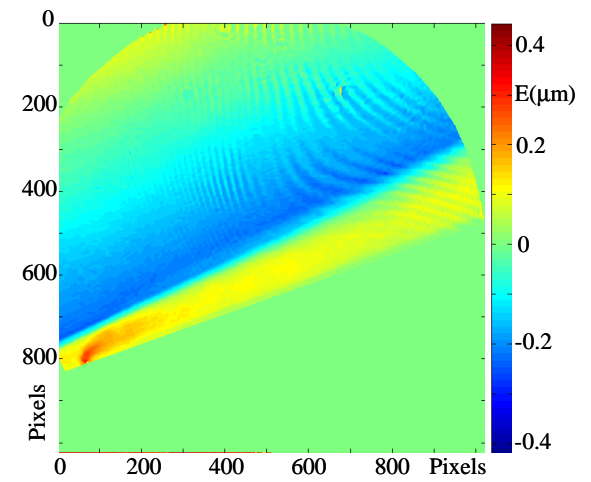

b) Optical thickness

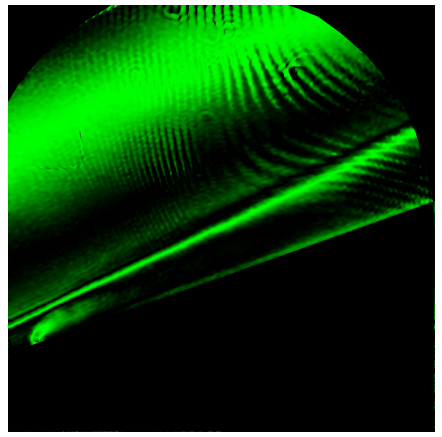

c) Luminous intensity

Fig. 4 Visualization of hydrogen jet.

\section{Comparisons with numerical simulations}

The wind-tunnel run studied in previous sections has also been numerically simulated at an instant of the run slightly earlier than for holographic visualization so that reservoir total pressure $(\mathrm{Pt})$ and enthalpy $(\mathrm{Ht})$ weakly differs: $\mathrm{Pt}=497 \mathrm{bar}$ and $\mathrm{Ht}=9.34 \mathrm{MJ} / \mathrm{kg}$ for the computation; $\mathrm{Pt}=472$ bar and $\mathrm{Ht}=8.70 \mathrm{MJ} / \mathrm{kg}$ for the visualization. Nevertheless, the measured Pitot pressures (P't) in the test section are very close: $\mathrm{P}$ 't $=27,058 \mathrm{~Pa}$ for the computation and $\mathrm{P}^{\prime} \mathrm{t}=27,844 \mathrm{~Pa}$ for the visualization.

\subsection{Numerical modeling}

3D Reynolds Average Navier-Stokes computations have been performed to simulate the flow. Only the flow on the upper part of the plate is computed. The geometry of the injector is not modeled; injection is simulated by a step velocity profile imposed on a disk boundary condition of $1 \mathrm{~mm}$ diameter. The mesh used is block-structured and contains approximately 10.4 million cells. The cell size at the injection area is $50 \mu \mathrm{m}$ and the height of the first cell above the wall is $20 \mu \mathrm{m}$.

Starting from the reservoir pressure and enthalpy, the flow is rebuilt inside the test section using the PNS code PANASCE. At $\mathrm{t}=35 \mathrm{~ms}$, the Mach number, static pressure, and static temperature in the test section are respectively: $9.58,246 \mathrm{~Pa}$ and $471 \mathrm{~K}$. These values are used as input for the RANS computation. The computed unit Reynolds number inside the test section is $274,262 \mathrm{~m}^{-1}$. Hydrogen is injected at Mach 1with a total pressure of 17.4 bar and a total temperature of $300 \mathrm{~K}$. Computations are 
run with the ONERA CEDRE code. Turbulence is taken into account using a k-1 model. Combustion is modeled by a quasi-laminar approach: terms of species production are calculated by the Arrhenius law based on the Eklund kinetics, 1990. Eulerian fluxes are evaluated at cells faces using a Roe scheme. Time integration is performed by an implicit Euler scheme of first order accuracy. Second order in space is achieved by a MUSCL type interpolation on cells faces.

\subsection{Description of the flow and comparison with experiment}

Principal features of the flow have been described by Schetz et al. [7] and Viti [8] and are gathered on Fig. 5 which focus on the injection. The jet expends through an expansion fan and is re-compressed through a barrel shock whose windward part is folded by the transverse main flow. The bow shock created in front of the injection intersects the plate leading edge shock. The adverse pressure gradient induced by this bow shock causes boundary layer separation, which results in a characteristic $\lambda$ shape shock pattern. The barrel shock ends with a Mach disk after which flow is subsonic. This subsonic flow is then re-accelerated by surrounding flow through mixing layers. Another mixing layer takes place between the bow shock and the barrel shock. Even if the detail of the flow is not captured by holographic visualization, the interaction between the leading-edge shock and the injection bow shock is clearly visible on Fig. 5 and 6. On Fig. 6, the schlieren visualization obtained from holographic interferogram is compared to a "numerical' schlieren computed from simulation, in the plane of symmetry. Due to the fact that the numerical schlieren is calculated in the symmetry plane, the leadingedge shock cannot be captured downstream of its interaction with the jet bow shock. For the same reason, the bow shock itself is more discernible than in the holographic based visualization. Nevertheless, the comparison between the two approaches show a very good agreement on the location of the shocks interaction, and of the downstream position of the bow shock.

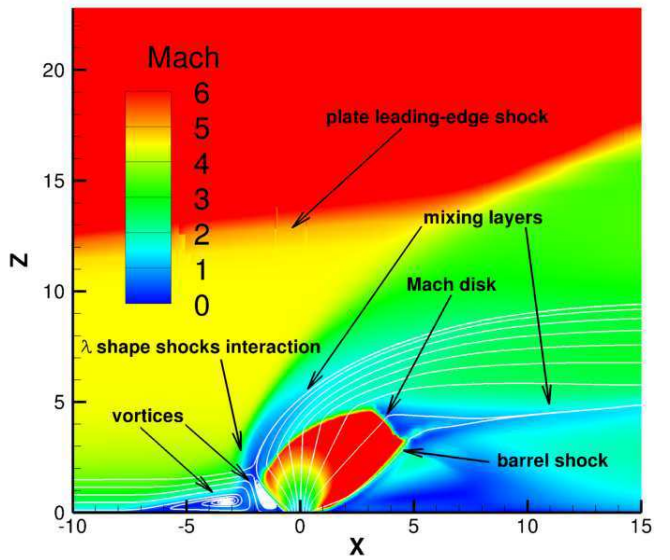

Fig. 5: 2D view in the symmetry plane of the flow structure near the injection

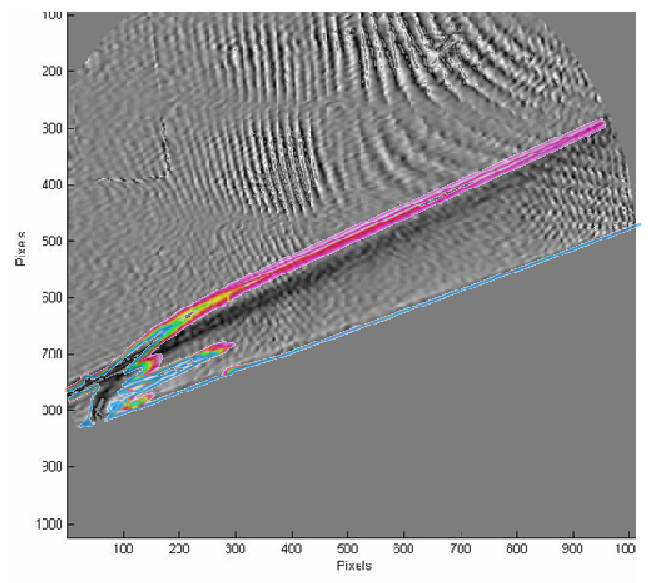

Fig.6: Comparison of Schlieren visualizations calculated from holographic interferogram and from numerical simulation.

\section{Conclusion}

As a conclusion, some improvements can be brought to this optical setup. As the resolution of digital holographic interferometry depends mainly on the pixel size and the pixel number of the CCD or CMOS sensor, the interferograms have to be recorded with a camera having a high spatial resolution 
sensor and a high framing rate. In order to increase the spatial carrier frequency, the wavelength of the green DPSS laser can be replaced by a blue DPPSS laser having an adapted coherence length. The sensitivity of the measurement can be increased by changing the focal length of the large achromatic lens $(800 \mathrm{~mm})$ and the optical bench has to be isolated from external vibrations.

Anyway, the feasibility of digital holographic interferometry has been demonstrated in the F4 hypersonic wind tunnel. The phase difference maps have led to obtain the optical path difference or thickness maps. If the flow is assumed to be two-dimensional in the spanwise direction of the flat plate, the refractive index maps of the phenomenon could be obtained from the optical thickness maps.

The results obtained in digital holographic interferometry can be used for several interesting comparisons. From the phase difference maps, the holographic images and schlieren images in the $\mathrm{x}$ and y directions can be recalculated, which is not possible with a conventional schlieren optical setup. Finally, the feasibility of the method has shown the wide possibilities of the technique and it gives access to the quantitative measurements of the flow as the optical thickness and the refractive index under particular conditions. In addition, if the characteristics of the ionized gas are well known, the value of the gas density upstream and in the shock layer can be estimated.

\section{Acknowledgment}

This research is partially funded by the French National Agency for Research (ANR) under grant agreement $n^{\circ}$ ANR 2010 BLAN 0302 and the author wishes to thank Sébastien Defort et Laurent Serre finding additional funding for tests and calculations.

\section{References}

1. Naydenova I. Advanced Holography - Metrology and Imaging. 1st edition, InTech Open Access, 2011.

2. Desse JM and Picart P. Digital color holography applied to fluid and structural mechanics. Opt. \& Las. in Engineering, Vol. 50, pp 18-28, 2012.

3. Surget J, Philbert M and Bize D. Schlieren device and holographic interferometer for hypersonic flow visualization, Proc. of 6th Intl. Symp. on flow visualization, Yokohama (Japan), 1992.

4. Surget $\mathbf{J}$ and Dunet G. Multipass holographic interferometer for the high enthalpy hypersonic wind tunnel F4, Advanced research workshop on "New trends in instrumentation for hypersonic research, Le Fauga, France, 1992.

5. Smith J, Jones B and Brown J. Digital color holography applied to fluid and structural mechanics. Opt. \& Las. in Engineering, Vol. 50, pp 18-28, 2012.

6. Desse JM, Picart P and Tankam P. Digital three-color holographic interferometry for flow analysis, Optics Express, Vol. 16, nº 8, pp. 5471-5480, 2008.

7. Schetz JA, Hawkins PF and Lehman H. Structure of highly underexpanded transverse jets in a supersonic stream, AIAA journal, Vol. 5, n 5, pp.882-884, 1966.

8. Viti V, Neel R and Schetz JA. Detailed flow physics of the supersonic jet interaction flow field, Physics of Fluids, Vol. 21, n 4, 046101, pp.1-16, 2009.

\section{Copyright Statement}

The authors confirm that they, and/or their company or institution, hold copyright on all of the original material included in their paper. They also confirm they have obtained permission, from the copyright holder of any third party material included in their paper, to publish it as part of their paper. The authors grant full permission for the publication and distribution of their paper as part of the ISFV16 proceedings or as individual off-prints from the proceedings. 